Rot ary echoes due to magnet i c di pol ar i nt er acti on i $n$ a mil $t i-$ level NM system

\begin{tabular}{|l|l|}
\hline 著者 & Hat anaka H r oshi \\
\hline $\begin{array}{l}\text { j our nal or } \\
\text { publ i cat i on t i t l e }\end{array}$ & Physi cs Let ters A \\
\hline vol une & 112 \\
\hline number & 9 \\
\hline page $r$ ange & $471-473$ \\
\hline year & $1985-11-18$ \\
\hline URL & ht t p: //hdl . handl e. net /2297/11659 \\
\hline
\end{tabular}


Rotary Echoes Due to Magnetic Dipolar Interaction in a Multi-Level NMR System

\section{H. HATANAKA}

Department of Physics, Faculty of Education, Kanazawa University, Kanazawa, Japan

A new type of rotary echo which is due to magnetic dipolar interaction has been observed in a multi-level NMR system. From the echo-envelope decay we can estimate a second moment due to a part of dipolar interaction participating only in the resonant transition. 
Rotary echoes [1] are known as recovery phenomena of transient nutation [2] damped owing to an intensity distribution of the effective field. In the present paper, we report a new type of rotary echo produced by reversing the contribution of magnetic dipolar interaction in a multi-level NMR system. The non-resonant levels contribute to the echo formation.

In order to illustrate the mechanism of the echo formation, we consider the same NMR system as in a previous work [3], that is, a multi-level system of quadrupolar nuclei which is subjected to a static magnetic field $H_{0}$ applied along the principal axis ( $z$-axis) of the electric field gradient tensor. The electric quadrupole coupling energy is much smaller than the Zeeman energy $\hbar \gamma H_{0}, \quad$ where $\gamma$ is the gyromagnetic ratio.

Let us assume that an $\mathrm{rf}$ field $2 H_{1} \cos \Omega t$, which is applied along the $x$-axis, selectively excites one transition $I_{z}=m \longleftarrow \longrightarrow m$ - 1 with resonance frequency $\omega$, where the angular frequency $\Omega$ is slightly different from $\omega$ by $\delta \quad(\delta=\Omega-\omega)$. While the rf field is on, the resonant spins are subjected to the effective field $H_{\mathrm{e}}=\sqrt{\delta^{2}+\beta^{2}} / \gamma$ which makes an angle $\theta=\cos ^{-1}\left(\delta / \gamma H_{\mathrm{e}}\right)$ with the $z$-axis in the reference frame rotating with the angular frequency $\Omega$, where $\beta=\gamma H_{1}<m\left|I_{+}\right| m-1>$. If the effective field is much larger than the local field due to the magnetic dipolar interaction, the decay of the transient nutation is governed by the time-independent part $\mathrm{H}_{d}^{\dagger}$ of the dipolar interaction Hamiltonian in the doubly rotating frame, the frame rotating about the effective field with angular frequency $\gamma H_{\mathrm{e}}[4]$. The Hamiltonian $\mathrm{H}_{\mathrm{d}}^{\dagger}$ is derived from the 
truncated dipolar interaction Hamiltonian in the multi-level system [5]. As shown in the previous paper [3], $\mathrm{H}_{\mathrm{d}}^{\dagger}$ is complicated but is divided into three parts as

$$
\mathrm{H}_{\mathrm{d}}^{\dagger}=\mathrm{H}_{\mathrm{d} 1}^{\dagger}+\mathrm{H}_{\mathrm{d} 2}^{\dagger}+\mathrm{H}_{\mathrm{d} 3}^{\dagger}
$$

$\mathrm{H}_{\mathrm{d} 1}{ }^{\dagger}$ participates only in the resonant transition $\mathrm{m} \leftarrow \rightarrow \mathrm{m}-1 . \quad \mathrm{H}_{\mathrm{d} 2}{ }^{\dagger}$ and $\mathrm{H}_{\mathrm{d} 3}{ }^{\dagger}$ show the contributions from the non-resonant levels, and $\mathrm{H}_{\mathrm{d} 3}{ }^{\dagger}$ commutes with fictitious spin-1/2 operators $S_{X}, S_{Y}$ and $S_{Z}$ associated with the transition $m \leftarrow \rightarrow m-1[3,6]$, where $\vec{S}=\sum_{k} \vec{S}_{k}$, and $X, Y$ and $Z$ indicate the axes in the tilted rotating frame with the $Z$-axis along the effective field and the $Y$-axis which coincides with the $y$-axis.

If the fictitious spins are rotated about the $X$-axis through an angle $\pi$, the Hamiltonian $\mathrm{H}_{\mathrm{d}}^{\dagger}$ is transformed to

$$
U \mathrm{H}_{\mathrm{d}}^{\dagger} U^{-1}=\mathrm{H}_{\mathrm{d} 1}^{\dagger}-\mathrm{H}_{\mathrm{d} 2}^{\dagger}+\mathrm{H}_{\mathrm{d} 3}^{\dagger}
$$

where $U=\exp \left(i \pi S_{X}\right)$. It is noted that the sign of $\mathrm{H}_{\mathrm{d} 2}{ }^{\dagger}$ is changed. Therefore, it is expected that the $\pi$-rotation of the fictitious spins recovers the decay of the transverse magnetization due to $\mathrm{H}_{\mathrm{d} 2}{ }^{\dagger}$ in the tilted rotating frame and gives rise to a rotary echo, though the recovery is not complete. If the $\pi$-rotation is carried out at $t=\tau$, the time development of the normalized magnitude of the transverse magnetization becomes 


$$
\Gamma(t)=1-\frac{1}{2} M_{2}^{(1)} t^{2}-\frac{1}{2} M_{2}^{(2)}(t-2 \tau)^{2}+\ldots \ldots, \quad(t>\tau),
$$

where $M_{2}^{(1)}$ and $M_{2}^{(2)}$ represent the second moments of the absorption lines in the rotating frame which are broadened by $\mathrm{H}_{\mathrm{d} 1}^{\dagger}$ and $\mathrm{H}_{\mathrm{d} 2}{ }^{\dagger}$, respectively. As is expected the effect of $M_{2}{ }^{(2)}$ is cancelled at $t=2 \tau$. This type of echo is not expected to be observed at exact resonance since $\mathrm{H}_{\mathrm{d} 2}^{\dagger}$ vanishes at $\delta=0$.

The experiment was carried out at $13 \mathrm{MHz}$ on ${ }^{27} \mathrm{Al}$ nuclei $(I=5 / 2)$ in a single crystal of $\mathrm{Al}_{2} \mathrm{O}_{3}$ at room temperature. The echo formation was confirmed by observing the oscillatory behavior of the population difference in the transition $(-3 / 2)$ to $(-1 / 2)$ under the condition of $\delta=$ $\beta$, where the effective field tilts from the $z$-axis by an angle $\pi / 4$. The pulse sequence used for this experiment is illustrated in Fig. 1. An rf pulse with frequency $\Omega$ is applied along the $x$-axis at $t=0$. The $\pi$ -rotation about the $X$-axis is achieved by a $\pi$-phase shift of the $\mathrm{rf}$ carrier persisting for a time $\pi / \gamma H_{\mathrm{e}}$. The population difference $w(t)$ is measured by applying a $\pi / 2$-pulse with frequency $\omega$ after the first pulse is turned off at time $t$. The time interval of $500 \mu \mathrm{s}$ is introduced between the pulses to remove transients caused by the first pulse. The effect of spin-lattice relaxation can be neglected since the duration of the pulse sequence is very short compared with the relaxation time.

The experimental result is shown in Fig. 2. The upper trace was observed without the $\pi$-rotation, showing the usual transient nutation. The lower trace shows the echo signal obtained by making the $\pi$-rotation at $t=\tau=200 \mu \mathrm{s}$. The duration of the $\pi$-rotation is eliminated in 
the figure. The downward shift of the base line of the oscillation is due to the inversion of the nutation cone. The large shift of the echo-peak from $t=2 \tau$ is understood as a result of the incomplete recovery of the transverse magnetization.

Open circles in Fig. 3 show the echo-envelope decay obtained by plotting the oscillation-amplitude of $w(t)$ at $t=2 \tau$ as a function of $\tau$. Closed circles in the figure indicate the nutation decay $\Gamma(t)$.

The $\pi$-rotation may also produce ordinary rotary echoes due to the intensity-distribution of the effective field [7]. In order to confirm that the dipolar interaction is responsible for this echo formation, we compare the echo-envelope decay with the nutation decay $\Gamma_{\mathrm{h}}(t)$ in the absence of the intensity distribution of the effective field. If the dipolar interaction does not participate in the echo formation, the echo-envelope decay should coincide with $\Gamma_{\mathrm{h}}(t)$.

$\Gamma_{\mathrm{h}}(t)$ was obtained by the following procedure. We assume that the observed nutation decay $\Gamma(t)$ can be written as

$$
\Gamma(t)=\Gamma_{\mathrm{i}}(t) \Gamma_{\mathrm{h}}(t),
$$

where $\Gamma_{\mathrm{i}}(t)$ represents the nutation decay due only to the intensity distribution of the effective field. In order to estimate $\Gamma_{\mathrm{i}}(t)$, we carried out a similar experiment on ${ }^{19} \mathrm{~F}$ nuclei $(I=1 / 2)$ in a Teflon sample of the same size as that of the $\mathrm{Al}_{2} \mathrm{O}_{3}$ sample. From the observed nutation decay $\Gamma(\mathrm{F})(t)$ and the echo-envelope decay on ${ }^{19} \mathrm{~F}$ nuclei, the latter can be regarded as $\Gamma_{\mathrm{h}}(\mathrm{F})(t)$, we obtained $\Gamma_{\mathrm{i}}(t)$. The decay function $\Gamma_{\mathrm{h}}(t)$ for 
${ }^{27} \mathrm{~A} 1$ nuclei can be obtained from $\Gamma(t)$ and $\Gamma_{\mathrm{i}}(t)$ and the result is shown by the dashed line in Fig. 3.

From the fact that the decay curve shown by open circles is quite different from the dashed line, we can recognize that the contribution of the intensity distribution of the effective field to the rotary echo is negligible. From the echo-envelope decay near $t=0$, we can estimate the second moment $M_{2}^{(1)}$, since the second moment $\mathrm{M}_{2}{ }^{(2)}$ does not contribute to the echo-envelope decay.

As shown in the previous paper [3], the second moment $M_{2}^{(1)}$ vanishes when $\cos \theta=1 / \sqrt{3}$, which is the same as the well-known magic-angle condition [8]. Therefore, it is interesting to observe the echo-envelope decay under this condition, where the second moment due to the dipolar interaction vanishes. Figure 4 shows the rotary echo observed at $\theta=\cos ^{-1}(1 / \sqrt{3})$, where the $\pi$-rotation is performed at $t=$ $300 \mu \mathrm{s}$. In this case the intensity of the $\pi$-rotation rf-field is reduced to one half the intensity of the main one. As is expected the echo-envelope decay is lengthened. A detailed study is in progress.

The author thanks Mr. K. Hayashi for his assistance in instrumentation. 
References

[1] I. Solomon, Phys. Rev. Lett. 2 (1959) 301.

[2] H.C. Torrey, Phys. Rev. 76 (1949) 1059.

[3] H. Hatanaka, H. Deguchi and T. Hashi, J. Phys. Soc. Japan 54 (1985) 374.

[4] D. Wolf, Spin-temperature and nuclear-spin relaxation in matter (Oxford Univ. Press, London, 1979).

[5] H. Hatanaka and T. Hashi, J. Phys. Soc. Japan 50 (1981) 3629.

[6] A. Abragam, The Principles of Nuclear Magnetism (Oxford Univ. Press, London, 1961).

[7] H. Kessemeier and W.K. Rhim, Phys. Rev. B5 (1972) 761.

[8] W.I. Goldburg and M. Lee, Phys. Rev. Lett. 11 (1963) 255; M. Lee and W.I. Goldburg, Phys. Rev. 140 (1965) A1261. 
Figure Captions

Fig. 1. Pulse sequence used for observing the rotary echo due to magnetic dipolar interaction under the off-resonance condition of $\delta=$ $\beta$.

Fig. 2. Rotary echo due to magnetic dipolar interaction observed at $\delta=$ $\beta$. The upper trace shows the usual transient nutation. The lower trace, showing the rotary echo, was observed after the $\pi$-rotation of the spins carried out at $t=200 \mu \mathrm{s}$. The downward shift of the base line of the oscillation indicates the inversion of the nutation cone. The large shift of the echo-peak from $t=400 \mu \mathrm{s}$ is due to the incomplete recovery.

Fig. 3. Rotary echo envelope decay (open circles). The echo envelope decay is obtained by plotting the oscillation amplitude of $w(t)$ at $t=2$ $\tau$ as a function of $\tau$. Dashed line represents the nutation decay in the absence of the intensity distribution of the effective field, which is obtained by the aid of the similar experiment on ${ }^{19} \mathrm{~F}$ nuclei in Teflon. Closed circles show the observed nutation decay. These decay curves are normalized.

Fig. 4. Rotary echo due to magnetic dipolar interaction observed at $\theta=\cos ^{-1}(1 / \sqrt{3}) \quad(\sqrt{2} \delta=\beta) . \quad$ The $\pi$-rotation is performed at $t=300$ $\mu \mathrm{s}$. 


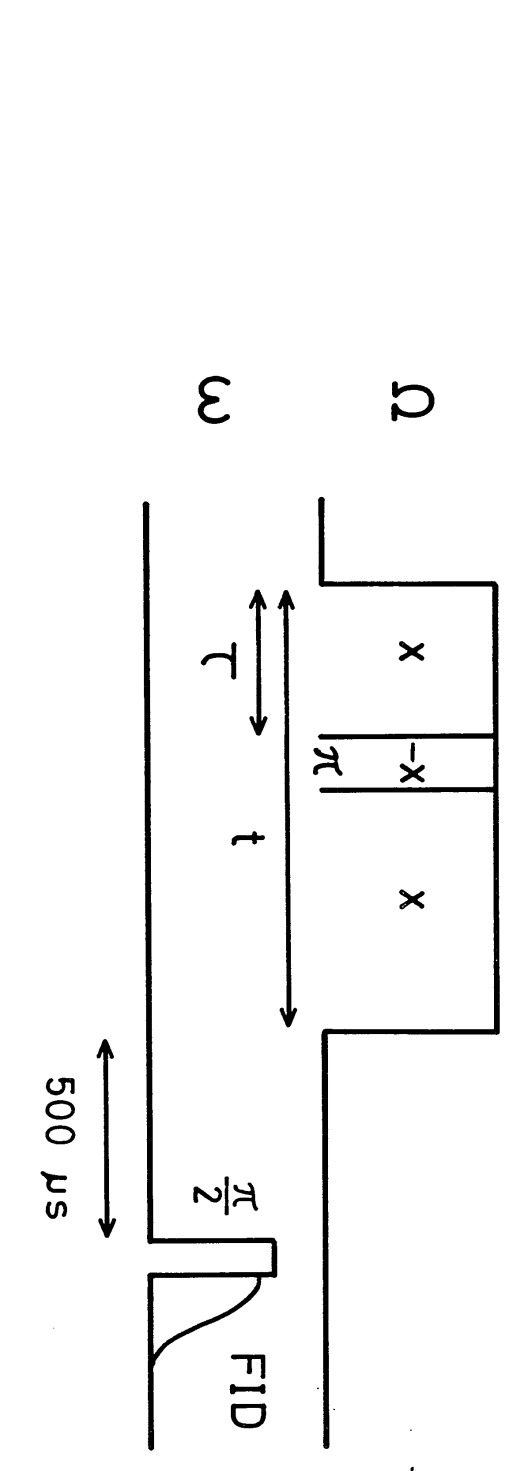




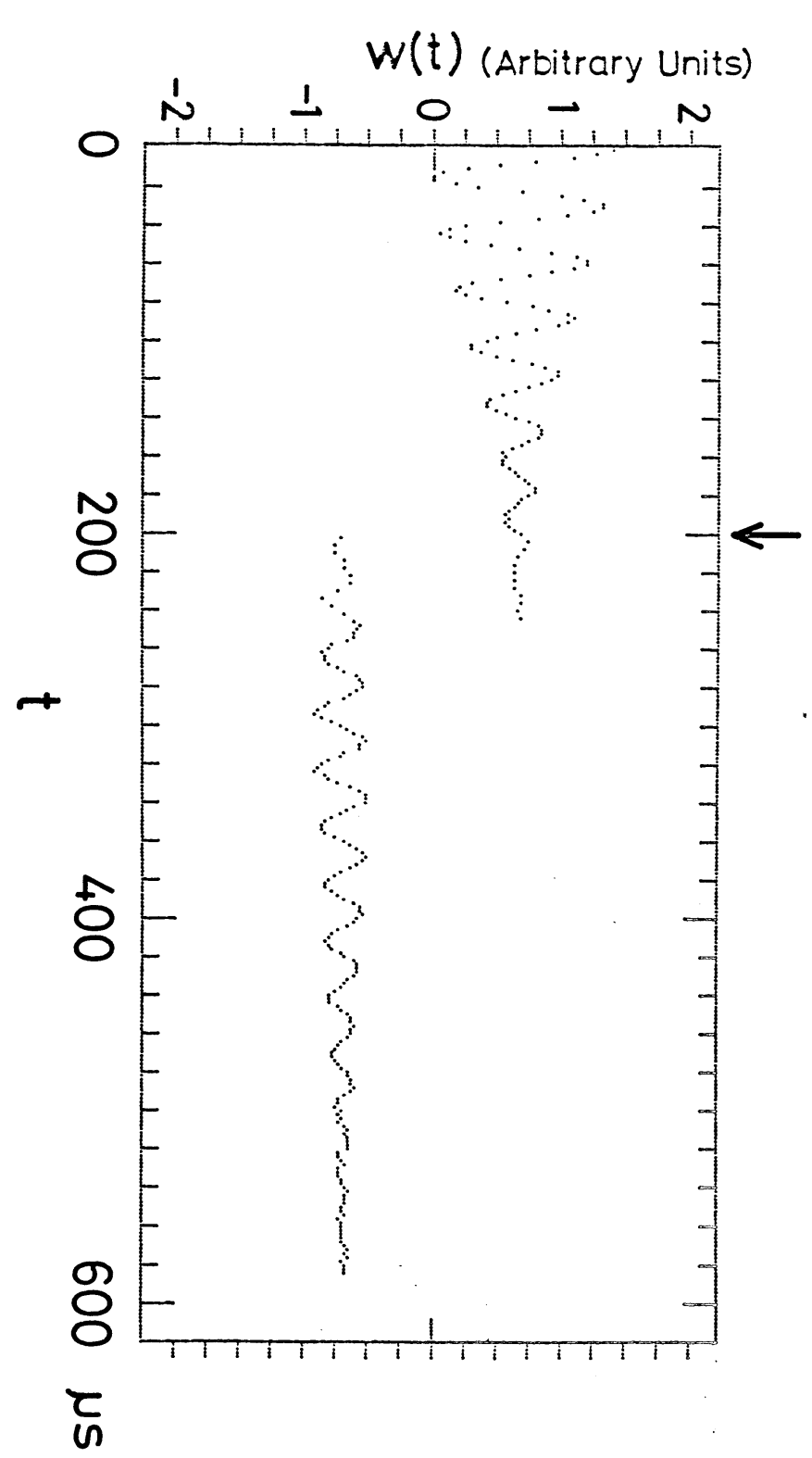




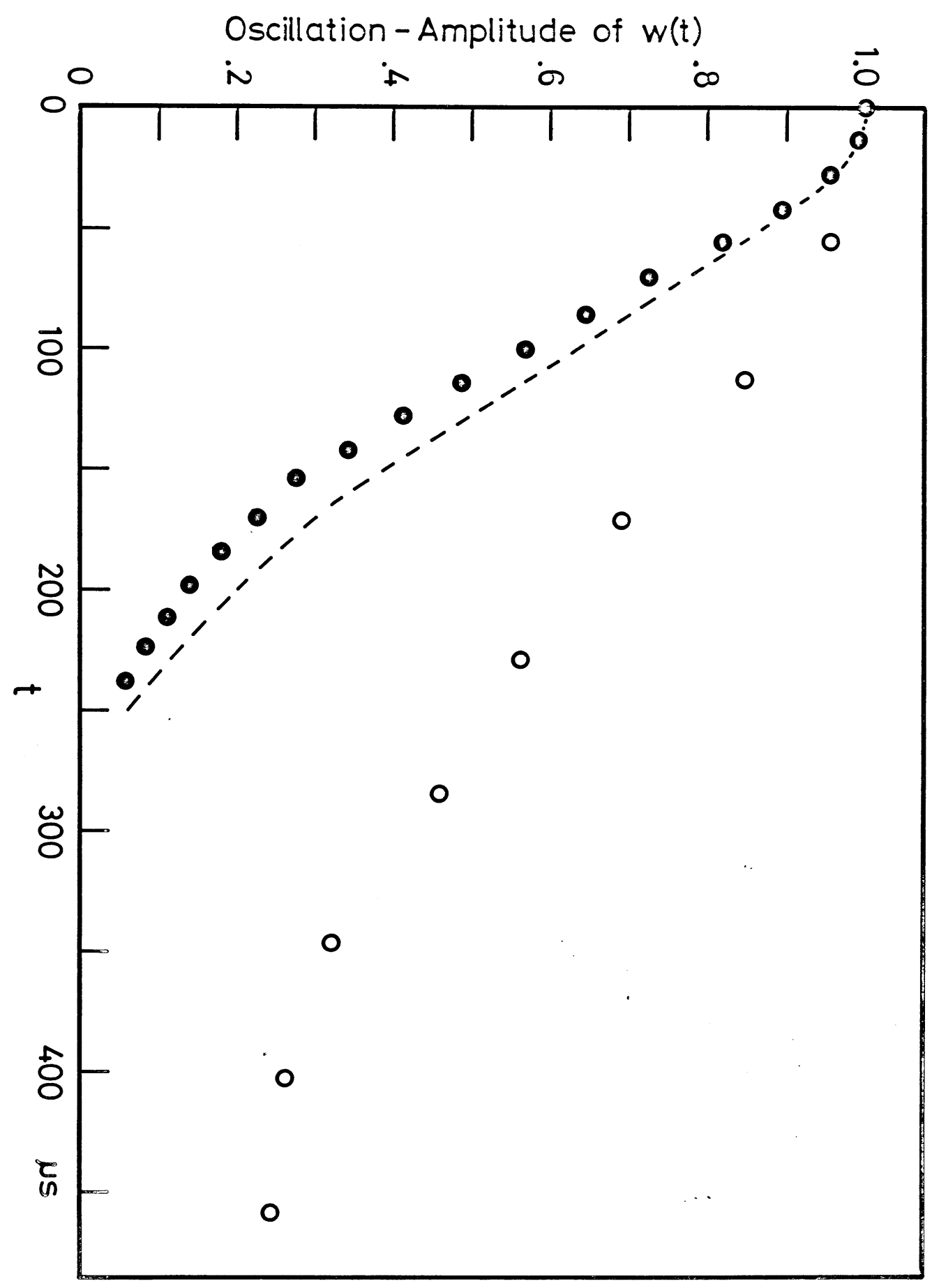




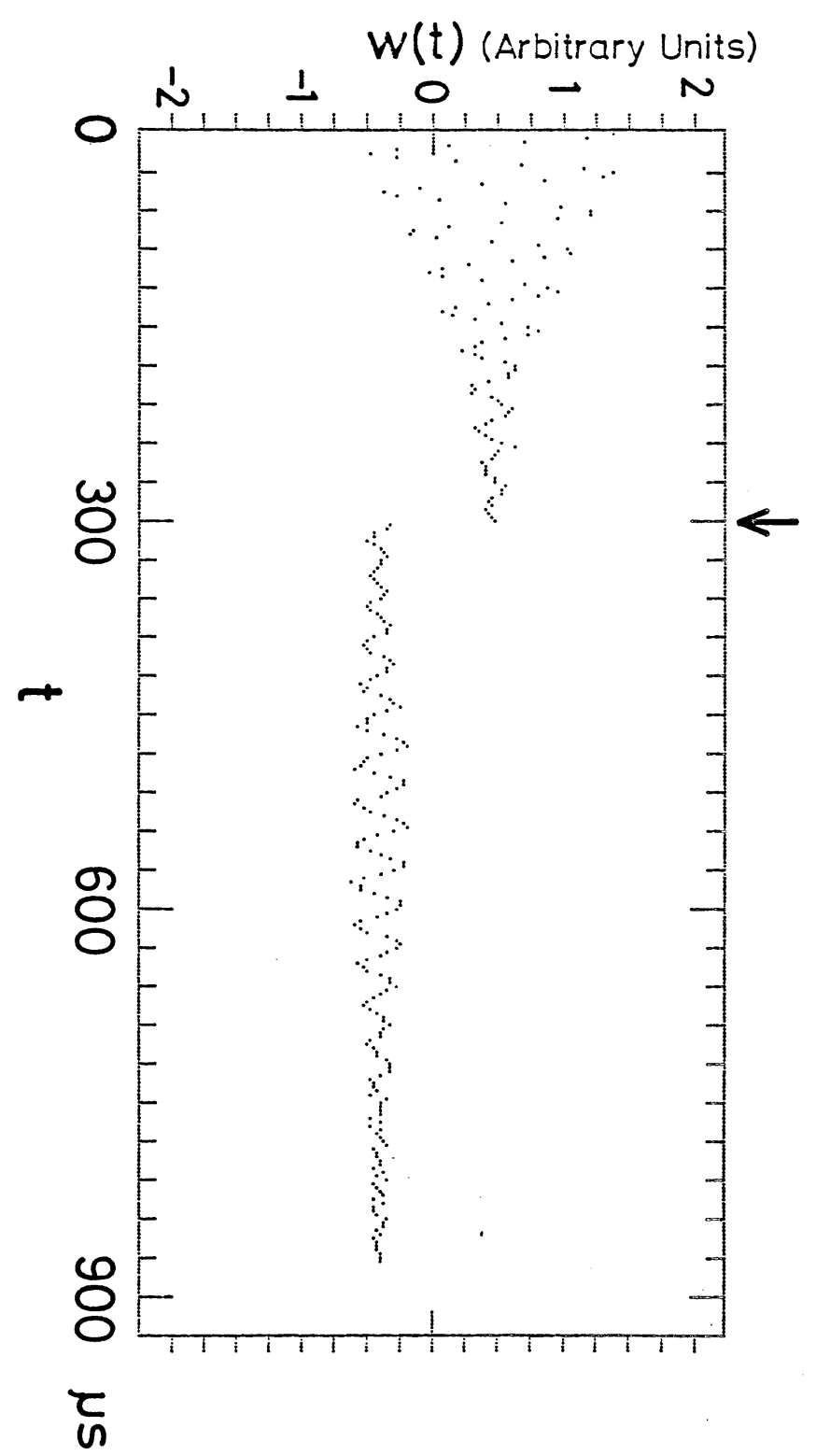

